\title{
Correlation of plasma protein from MDS, young and elderly patients by SDS-page
}

\section{Nathanielly de Lima Silva ${ }^{1}$, Danilo Nobre ${ }^{1}$, Josiel Nascimento dos Santos ${ }^{2}$, Márcia Santos Rezende ${ }^{1}$, Lúcio Henrique Sousa Pinheiro ${ }^{1}$, Carlos Arthur Cardoso Almeida ${ }^{2}$ and Dulce Marta Schimieguel ${ }^{1 *}$}

'Department of Pharmacy, Federal University of Sergipe, São Cristóvão, Sergipe, Brazil ${ }^{2}$ Institute of Pharmaceutical Science, Federal University of Alagoas, Maceió, Alagoas, Brazil

\section{Abstract}

Summary: Myelodysplastic Syndrome (MDS) is a heterogeneous group of clonal hematopoietic malignancies characterized by progressive cytopenias, ineffective hematopoiesis, bone marrow hypercellularity and transformation to acute myeloid leukemia (AML).

Objectives: Identify plasma proteins from MDS patients and from two healthy controls groups (young and elderly) by SDS-Page.

Methods: Plasma from 08 healthy young, 08 healthy elderly and 08 MDS patients were used for this study. Proteins were fractionated, precipitated, used for SDS-PAGE gel analysis, stained with comassie brilliant blue, scanned and bands were analyzed.

Results: It was possible to identify in both, $20 \%$ fraction and supernatant, proteins that were differentially expressed in each group. The ones that have showed some clinical relevance. Fibronectin was highly expressed only in the young control group. a2-Macroglobulin was also expressed in both control groups, but it was not expressed in the MDS group. Haptoglobin was highly expressed only in the elderly control and SMD groups.

Conclusion: Protein expression in plasma can be a biomarker for MDS, and may play a key role in the process of aging and hematologic malignancies development.

\section{More Information}

*Address for Correspondence: Dulce Marta Schimieguel, Department of Pharmacy, Federal University of Sergipe, São Cristóvão, Sergipe, Brazil, Tel: +55 79 3194-6319;

Email: dulcemarta@hotmail.com; hematox2017@hotmail.com

Submitted: 29 October 2019

Approved: 08 November 2019

Published: 11 November 2019

How to cite this article: Silva ND, Nobre D, dos Santos JN, Rezende MS, Pinheiro LHS, et al. Correlation of plasma protein from MDS, young and elderly patients by SDS-page. Int J Bone Marrow Res. 2019; 2: 087-088.

DOI: dx.doi.org/10.29328/journal.ijbmr.1001009 ORCiD: oricd.org/0000-0001-9668-1514 Copyright: () 2019 Silva ND, et al. This is an open access article distributed under the Creative Commons Attribution License, which permits unrestricted use, distribution, and reproduction in any medium, provided the original work is properly cited.

Check for updates

\section{Introduction}

Recently, medicine has been made great progress in the treatment of many types of cancer. On the other hand, MDS and AML remains one of the most unfavorable hematological malignancies with poor prognosis. Consequently, elderly MDS patients continue to present a great number of issues, which aging is one of the major prognostic factor $[1,2]$.

Most patients is diagnosed by the age of 60 , which the highest incidence is around 70 to 75 years old. Age is one of the main factors in the development of many types of cancer, as occur in MDS. Nonetheless, other risk factors may be evaluated with age, such as environmental, cellular and genetic factors [3].

MDS diagnosis includes quantitative and qualitative analysis of peripheral blood and bone marrow. Bone marrow biopsy, cytogenetics, molecular analysis and immunophenotyping by flow cytometry are used to confirm the diagnosis. However, usually it is difficult to elucidate MDS diagnosis even to the most expert professionals [4].

There are several molecular markers associated with different biological functions of this disease, but very few are effectively proteins. In fact, plasma proteomes of several different MDS subgroups have been investigated and compared with those of healthy donors, but the resulting data has not be yet clear enough to be useful for the selection and proposal of diagnostic biomarker candidates [5].

\section{Material and Methods}

The Human Ethics Committee of the Federal University of Sergipe study approved protocol: 3.225.938. A total of 24 subjects, all females, were separated into three groups: 08 
healthy young, age ranged from 20 to 30 years old named the Young Group; 08 healthy elderly, age ranged from 60 to 80 years old, named the Elderly Group and 08 SMD patients, age ranged from 19 to 86 years old, named the SMD Group.

From all subjects and controls, it was collected $10 \mathrm{~mL}$ of blood in EDTA tube. Plasma was separated and frozen at $-80^{\circ} \mathrm{C}$ until analysis. Proteins were fractionated and precipitated. The $20 \%$ fraction and the supernatant were used for SDS-PAGE gel analysis. Samples were quantified [6], (Bradford method) and a $30 \mathrm{ng}$ were applied to a $10 \%$ polyacrylamide gel. Gel run for $1,5 \mathrm{~h}$ at $100 \mathrm{v}$, and later, was stained with Comassie brilliant blue G-250 a 0,2\%, scanned and bands were analyzed.

\section{Results}

Protein screening by SDS-PAGE presented relevant results, showing that it was possible the identification of proteins expressed in different ways in plasma of the 03 groups analyzed.

It was possible to identify in both, $20 \%$ fraction and supernatant, proteins that were differentially expressed in each group. The ones that have showed some clinical relevance. Fibronectin was highly expressed only in the young control group. $\alpha 2$-Macroglobulin was also expressed in both control groups, but not in the MDS group. Haptoglobin was highly expressed only in the elderly control and MDS groups, according to figure 1 .

\section{Discussion}

Fibronectin is a plasma protein normally related in several different biological functions, including cell adhesion, growth, migration and differentiation, which justify its high expression in young adults compared to elderly. In cancer and other pathological situations, fibronectin shows a decrease in expression, as well as an increase in the degradation, this is also shown in these results. Besides its multiple function, $\alpha 2$ macroglobulin is one of the major plasma antiproteinases. It

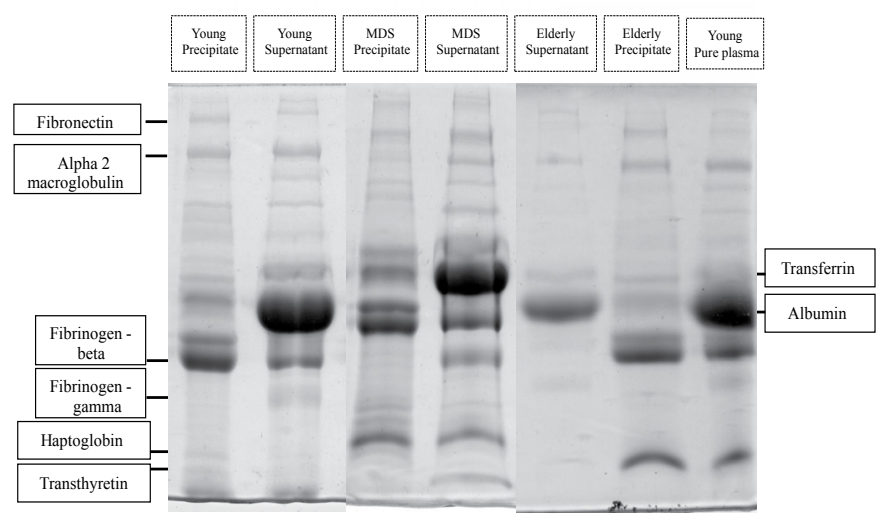

Figure 1: Coomassie brilliant blue stained SDS-PAGE gel.

Young Precipitate: Plasma pool of young individuals, $20 \%$ precipitated $(\mathrm{NH} 4) 2 \mathrm{SO} 4$; Young Supernatant: Plasma pool of young individuals, supernatant left after $20 \%$ precipitation ( $\mathrm{NH} 4)$ 2SO4; MDS Precipitate: Plasma pool of patients with suspected MDS, 20\% precipitated (NH4) 2SO4; MDS Supernatant: Plasma pool of patients with suspected MDS, supernatant left after $20 \%$ precipitation (NH4)04; Elderly Precipitate: Plasma pool of elderly individuals, $20 \%$ precipitate (NH4) 2SO4; Elderly Supernatant: Plasma pool of elderly subjects, supernatant left after $20 \%$ precipitation (NH4) 2SO4; Young Pure Plasma: Plasma pool of young individuals without (NH4) $2 \mathrm{SO} 4$ addition. plays also important role in inflammatory process by binding to several cytokines and/or receptors, and it has also been associated with aging and cancer. This may explain the results showed in this study. Haptoglobin is a tetrameric plasma protein that binds to hemoglobin with high affinity. It has a clinical importance and can be used both as a diagnostic and prognostic marker since the levels of haptoglobin increases/ decreases in various pathological conditions. Recently, a positive relationship was reported between the increase in haptoglobin and aging, compared to the reference value for adults, the haptoglobin concentration was found as low in the young. Results that are similar to what we have found here.

\section{Conclusion}

SGS-PAGE gel electrophoresis can be a cheap and powerful tool to discriminate protein expression in plasma fractions and precipitate from healthy and MDS patients; there are proteins that may play a key role in the process of aging and cancer, and thus may be possibly associated with MDS development. However, further studies must be done in order to conclude this.

\section{Acknowledgment}

The authors thank the professionals from the University Hospital of the Federal University of Sergipe e from the Hospital de Urgência de Sergipe, that was involved in this study.

\section{Financial Support}

This work was supported by Conselho Nacional de Desenvolvimento Científico e Tecnológico - CNPq (Proc. Proc. 306464/2016-0 and 423833/2018-9) and by Fundação de Apoio à Pesquisa e à Inovação Tecnológica do Estado de Sergipe (FAPITEC/SE).

\section{References}

1. Mueller BU, Seipel K, Pabst T. Myelodysplastic syndromes and acute myeloid leukemias in the elderly. Eur J Intern Med. 2018; 58: 28-32.

PubMed: https://www.ncbi.nlm.nih.gov/pubmed/30527920

2. DeZern AE, Malcovati L, Ebert BL. CHIP, CCUS, and Other Acronyms: Definition, Implications, and Impact on Practice. Am Soc Clin Oncol Educ Book. 2019; 39: 400-410.

PubMed: https://www.ncbi.nlm.nih.gov/pubmed/31099654

3. Zini G. Diagnostics and Prognostication of Myelodysplastic Syndromes. Ann Lab Med. 2017; 37: 465-474.

PubMed: https://www.ncbi.nlm.nih.gov/pubmed/28840983

4. Papaemmanuil E, Gerstung M, Malcovati L, Tauro S, Gundem G, et al. Clinical and biological implications of driver mutations in myelodysplastic syndromes. Blood. 2013; 122: 3616-3627. PubMed: https://www.ncbi.nlm.nih.gov/pubmed/24030381

5. Saha S, Halder S, Bhattacharya D, Banerjee D, Chakrabarti A. Fractional Precipitation of Plasma Proteome by Ammonium Sulphate: Case Studies in Leukemia and Thalassemia. J Proteomics Bioinformatics. 2012; 5.

6. Bradford MM. A rapid and sensitive method for the quantitation of microgram quantities of protein utilizing the principle of protein-dye binding. Analytical Biochemistry. 1976; 72: 248-254.

PubMed: https://www.ncbi.nlm.nih.gov/pubmed/942051 